\title{
Corticotropin releasing hormone receptor $C R H R 1$ gene is associated with tianeptine antidepressant response in a large sample of outpatients from real-life settings
}

\author{
Nicolas Ramoz $\mathbb{E}^{1}$, Nicolas Hoertel ${ }^{1,2,3}$, Bénédicte Nobile $\mathbb{E}^{4}$, Géraldine Voegeli, ${ }^{1,5}$, Ariane Nasr ${ }^{1}$, Yann Le Strat ${ }^{1,6}$,
} Philippe Courtet $\mathbb{D}^{4}$ and Philip Gorwood ${ }^{1,5}$

\begin{abstract}
Polymorphisms of genes involved in the hypothalamic-pituitary-adrenocortical (HPA) axis have been associated with response to several antidepressant treatments in patients suffering of depression. These pharmacogenetics findings have been reported from independent cohorts of patients mostly treated with selective serotonin reuptake inhibitors, tricyclic antidepressant, and mirtazapine. Tianeptine, an atypical antidepressant, recently identified as a mu opioid receptor agonist, which prevents and reverses the stress induced by glucocorticoids, has been investigated in this present pharmacogenetics study. More than 3200 Caucasian outpatients with a major depressive episode (MDE) from real-life settings were herein analyzed for clinical response to tianeptine, a treatment initiated from $79.5 \%$ of the subjects, during 6-8 weeks follow-up, assessing polymorphisms targeting four genes involved in the HPA axis (NR3C1, FKPB5, CRHR1, and AVPR1B). We found a significant association $(p<0.001)$ between CRHR1 gene variants rs878886 and rs16940665, or haplotype rs878886*C-rs16940665*T, and tianeptine antidepressant response and remission according to the hospital anxiety and depression scale. Analyses, including a structural equation model with simple mediation, suggest a moderate effect of sociodemographic characteristics and depressive disorder features on treatment response in individuals carrying the antidepressant responder allele rs8788861 (allele C). These findings suggest direct pharmacological consequences of CRHR1 polymorphisms in the antidepressant tianeptine response and remission, in MDE patients. This study replicates the association of the CRHR1 gene, involved in the HPA axis, with (1) a specificity attributed to treatment response, (2) a lower risk of chance finding, and in (3) an ecological situation.
\end{abstract}

\section{Introduction}

Major depressive disorder (MDD) is usually considered as being associated with a deregulation of the hypothalamic-pituitary-adrenocortical (HPA) axis. The hyperactivity of the HPA system might be testified by an elevated neurotransmission of the corticotropin-releasing

\footnotetext{
Correspondence: Nicolas Ramoz (nicolas.ramoz@inserm.fr)

${ }^{1}$ Université de Paris, Institute of Psychiatry and Neuroscience of Paris (IPNP),

INSERM U1266, Team Vulnerability of Psychiatric and Addictive Disorders, 75014 Paris, France

${ }^{2}$ Assistance Publique-Hôpitaux de Paris (APHP), Corentin Celton Hospital,

Department of Psychiatry, 92130 Issy-les-Moulineaux, France

Full list of author information is available at the end of the article
}

hormone (CRH/CRF) and arginine vasopressin (AVP) peptides, which stimulate the production of adrenocorticotropic hormone and cortisol, leading to a low-grade inflammation in patients with $\mathrm{MDD}^{1}$. Furthermore, the negative feedback is controlled by the glucocorticoid receptors (GR) and interacting proteins, like FKBP5 protein $^{2-5}$. Interestingly, ketamine (an NMDA antagonist) has been shown to significantly improve depression symptoms within patients with treatment-resistant depression, may be partly due to its action on inflammation $^{6,7}$. Those data support the hypothesis of HPA axis dysfunction within MDD patients and consequently the 
hypothesis of improving depression by acting on this system. Convergent evidence showed that antidepressant treatments may normalize or modulate the abnormal functionality of the HPA system in MDD patients ${ }^{8-11}$. It seems legitimate to suppose that response to antidepressant treatment could be different according to antidepressant type and genetic polymorphisms of the HPA axis.

Accordingly, several polymorphisms of genes, involved in HPA axis, have been associated with response to several antidepressant treatments. These results of pharmacogenetics were observed for several polymorphisms of FKBP5 gene (which encodes a chaperon protein to GR) associated with clinical response in European Caucasian MDD patients to various antidepressant treatments, including selective serotonin reuptake inhibitors (SSRI), tricyclic antidepressant (TCA), and mirtazapine (drug targeting serotonergic and noradrenergic receptors) ${ }^{12}$. The amino acid change N363S in the glucocorticoid receptor, encoded by the single polymorphism nucleotide (SNP) rs56149945/rs6195 in the NR3C1 gene, was investigated among 367 depressed patients having received either SSRI, TCA, or mirtazapine ${ }^{13}$. This NR3C1 variant was associated with early response after 2 weeks of treatment ${ }^{13}$. Another pharmacogenetics association was observed between three SNPs encompassing NR3C1 gene and response to escitalopram (SSRI) or nortriptyline (a norepinephrine reuptake inhibitor) among 760 patients of the Genome-based Therapeutic Drugs for Depression study (GENDEP study) ${ }^{14}$. Haplotypes of three variants of the $\mathrm{CRH}$ receptor type 1 CRHR1 gene were associated with an SSRI (fluoxetine) or a TCA (desipramine), in highly anxious Mexican-American patients ${ }^{15}$. The pharmacogenetics association of CRHR1 gene with response and remission was confirmed in the Sequenced Treatment Alternatives to Relieve Depression, STAR*D cohort, a sample of 1768 MDD patients treated initially by an SSRI (citalopram) ${ }^{16}$. In this study, polymorphisms in $\mathrm{CRH}$-binding protein gene $(C R H B P), \mathrm{CRH}$ receptor 2 gene (CRHR2), $C R H$ gene, and arginine vasopressin receptor 1A gene (AVPR1A) were also associated with treatment response or remission ${ }^{16}$.

We therefore have converging evidence that genes can be used to efficiently participate in the prediction of antidepressants response, and many of these genes are involved in the HPA regulation. Nevertheless, replication study was not systematic, although independent replication is considered as one of the most solid way to eliminate or, at least, reduce chance finding, especially for samples with modest size. Assessing various biomarkers predicting antidepressant response in a large meta-analysis, FKBP5 gene was indeed identified as the second gene having the largest effect size reaching a significant threshold ${ }^{17}$, testifying in favor of a potential use in clinical practice. Nevertheless, antidepressants have variable mechanisms of action, and other antidepressants (i.e. apart from SSRI, SNRI and tricyclics) are worth being analyzed for their pharmacogenetics factors. We also need to further distinguish the (direct) pharmacogenetics effects from (intermediate) clinical variables, as many are known to be involved in treatment response (such as number of past episodes, level of anxiety, and severity of depression). Indeed, a gene involved in the HPA axis could be associated with higher vulnerability to severe, comorbid, or chronic depressive disorder. All these traits are strongly related to poorer chances of treatment response ${ }^{18}$. Finally, if pharmacogenetic associations are replicated and not attributed to a clinical intermediate factor, it is important to assess if such information is predictive in "real life", meaning in a large cohort of usual outpatients (i.e. with few exclusion criteria) evaluated in an ecological situation.

Tianeptine is an antidepressant with structural similarities with TCAs, but with different pharmacological properties. Tianeptine is an atypical antidepressant because its mechanisms of action clearly challenge the monoamine hypothesis, triggering a cascade of cellular adaptations that leads to antidepressant response. Interestingly, tianeptine prevents and reverses the stress induced by glucocorticoids (through dendritic remodeling in CA3 pyramidal neurons in the hippocampus), and it increases dendritic length and branching of neurons in the amygdala ${ }^{19,20}$. Tianeptine is able to inhibit the dendritic remodeling caused by stress or glucocorticoids ${ }^{20}$. Finally, tianeptine has been shown to be a mu opioid receptor (MOR) agonist ${ }^{21}$ and its antidepressant effects may be mediated by this receptor ${ }^{22}$. This is the most interesting role of tianeptine since opioid system is known to partly regulate HPA axis. Indeed, an MOR antagonist would increase HPA axis activation ${ }^{23}$ and an MOR agonist would mediate this activation ${ }^{24}$. Clinical efficacy and tolerability of tianeptine have been demonstrated in depressed patients ${ }^{25}$. Our goal was thus to investigate the pharmacogenetics of tianeptine response by screening genes coding the proteins that play a major role in the HPA axis, in an ecological sample to test the predictive capacity of this set of HPA genes.

We therefore collected a sample of outpatients treated with tianeptine for a major depressive episode (MDE). More than 3200 Caucasian outpatients were analyzed for clinical response to tianeptine and assessed by the genotyping of different SNPs targeting several genes involved in the HPA axis.

\section{Materials and methods \\ Outpatients and clinical assessment}

We used a French network of psychiatrists and general physicians who already collected large samples of 
depressed outpatients ${ }^{26,27}$ to constitute a novel cohort of antidepressant treated patients, named GENESE. This time, we included in the research tools a device allowing to collect saliva in order to perform pharmacogenetic analyses. A total of 3771 outpatients were enrolled. For this pharmacogenetic study, we restricted the analyses to the 3212 Caucasians $(85.18 \%)$ with available DNA $(N=$ $3042,94.71 \%)$ to reduce the risk of stratification biases at the genetic level, due to the variability of the allelic percentages of genetic variants in different ethnic groups. The sample size of 3212 Caucasian outpatients was sufficient to detect a pharmacogenetics difference of $5 \%$ between the responder and non-responder groups.

GENESE is a large prospective, naturalistic cohort of French outpatients diagnosed with MDE and treated with tianeptine (from whom they were naïve for the present episode). Tianeptine was initiated for $79.5 \%$ of subjects ( $N=2419$ outpatients) and was prescribed as a switch from another antidepressant treatment for $18.8 \%$ of subjects $(N=573$ outpatients) while this information was missing for $1.7 \%(N=50)$. Dosage of tianeptine ranged between 12.5 and $37.5 \mathrm{mg} /$ day, according to prescription recommendations. All other concomitant treatments for current somatic problems or associated symptoms of depression (e.g., sleep and agitation) were permitted, based on clinical judgment (see section "Treatments"). At the first visit, practitioners validated the diagnosis of MDE according to DSM-IV criteria. The practitioners also collected demographic data, major depressive disorder history, lifetime suicide attempt and alcohol or substance dependence at the first visit during a face-to-face interview. Non-inclusion criteria were age under 18 years old, non-Caucasian ethnicity, alcohol and substance dependence, or any other psychiatric pathology from axis I other than current MDE. The study was performed according to French regulatory guidelines and current codes of Good Clinical Practice. This study was approved by a local independent ethical committee (Comité de Protection des Personnes Ile de France XI/CPPIDF11, \#08042). Each patient was informed about the aims and procedures of the study and provided written, signed consent.

Depression was assessed with a French version of the hospital anxiety and depression scale (HADS) 16 items at baseline V1. The second visit (V2) was followed in a window between 6 and 8 weeks and a novel evaluation of the HADS was done. Furthermore, outpatients made a self-evaluation of the HADS at V1 and V2 and at 15 and 30 days after the beginning of the treatment. This scale has a good performance assessing depression severity in both psychiatric and primary care patients, a good change-sensitivity ${ }^{28}$ and is simply to understand and use. Most factor analyses found a two-factor solution in accordance with the Anxiety (HADS-A) and Depression (HADS-D) subscales.

\section{Phenotype definition of treatment response}

A positive treatment response (Resp + ) was defined as the reduction of at least $50 \%$ of the HADS score at V2 compared to V1. Remission of depression was defined as a HADS score lower than a cutoff of 8 at V2. Finally, an early partial response was defined with a difference of reduction of the HADS higher than 25\% after 2 weeks according to the report of the outpatient.

\section{Treatments}

Associated treatments that may have an impact on treatment response (23 with antiparkinsonian drugs, 24 with corticosteroids, and 152 with thyroid hormones) were also recorded, but they had the same frequency in both response groups $(p>0.186)$.

On the other hand, $2.1 \%(N=62)$ of outpatients also received a mood stabilizer, $2.7 \%(N=83)$ a mood stabilizer and benzodiazepine, $50.1 \%(N=1525)$ only benzodiazepine, and $45.1 \%(N=1372)$ no mood stabilizer nor benzodiazepine. Patients with mood stabilizers, benzodiazepines, or both, had lower chances of treatment response, respectively, $39.3 \%, 41.6 \%$, and $41.2 \%$ were responders versus $44.6 \%$ without $\left(\mathrm{Chi}^{2}=7.152\right.$, d.f. $=1$, $p=0.007)$.

The average dosage of tianeptine was not equivalent in responders and non-responders, more patients with lower dosages being in the group of non-responders. Because the range of dosage was high (between 12.5 and $75 \mathrm{mg}$ per day) and because the majority of patients were treated in accordance with guidelines (i.e. $37.5 \mathrm{mg}$ a day for more than $82 \%$ in both groups), later analyses systematically checked the initial results restricting the analyses to patients treated with the recommended dosage of $37.5 \mathrm{mg} /$ day.

\section{SNPs and genotyping}

Genomic DNA from outpatients was extracted according to the protocol of the manufacturer from salivary sample collected with Oragene DNA kit (DNA Genotek Inc). A total of 16 SNPs encompassing four pharmacogenetics candidate genes (NR3C1, FKPB5, CRHR1, and $A V P R 1 B$ ) were screened using TaqMan SNP genotyping assays on an Applied Biosystem 7900HT Fast Real-Time PCR System (Life Technologies).

Sixteen SNPs were selected in the Hapmap database of tagged SNPs that cover the haplotype blocks across the genes, according to associations with antidepressant response or MDD.

\section{Statistical analysis}

Hardy-Weinberg equilibrium tests, linkage disequilibrium $D^{\prime}$ and $r^{2}$ values and minor allele frequencies for the different SNPs were computed using Haploview 4.2 software. 
Categorical variables were presented as percentages, and quantitative variables as means with standard deviation. Comparisons of sociodemographic, clinical features, and genotype between patients' groups were performed using Student's $t$-test (normality was tested with Shapiro-Wilk test) for continuous variables and chi-square test for categorical variables. Furthermore, logistic regression was carried out for sociodemographic and clinical features as intermediate variables with CRHR1 SNP rs878886 in predictor for treatment response. General linear models were computed to generate risk factors associated with the positive treatment response in interaction with or without the CRHR1 SNP rs878886 response allele. Those analyses were performed with SPSS statistical software (version 23; IBM SPSS Statistics for Windows. Armonk, NY, IBM Corp.).

We used a structural equation model with simple mediation, which assumes that the effect of sociodemographic characteristics and depressive disorder features on treatment response is the same in people with and without rs8788861. This was done in the goal to examine whether differences in the prevalence of sociodemographic characteristics and depressive disorder features, associated with rs8788861, explain the greater response to tianeptine among individuals with this SNP (see Supplementary Materials \& Methods). All analyses were conducted in Mplus Version 7.2 (Muthen \& Muthen). Mplus provides estimates and tests of significance for direct and specific effects and total indirect effects. The default estimator for the analysis was the variance-adjusted weighted least-squares, a robust estimator appropriate for categorical variables.

\section{Results}

Treatment response to antidepressant in outpatients with an MDE

A total of 3212 Caucasian outpatients were treated by tianeptine for an MDE (Table 1), including a majority of women (62.5\%). At baseline (V1), the mean of HADS score was 27.8 and was decreased to 15.42 at the second visit (V2) after $43 \pm 13$ days.

Tianeptine was a treatment initiated in $79.5 \%$ of the MDE outpatients $(N=2419)$. A total of $42.8 \%$ outpatients were responders to tianeptine treatment. For the group of responders, the baseline of HADS score was 29.6 and decreased to 9.3 at V2, while in the group of non-responders, the V1 HADS score was 26.5 and decreased only to 20 . MDE outpatients with mood stabilizers, benzodiazepines, or both were responders (respectively, 39.3\%, 41.6\%, and $41.2 \%$ ) but they have significantly lower chances of treatment response versus $44.6 \%$ responders without mood stabilizer or benzodiazepines $(p=0.007)$.
Sex ratio was not different between responders and non-responders (Table 1). Responders were significantly younger than non-responders $(48.04 \pm 14.50$ versus $50.50 \pm 15.02$ years), and they had a higher educational level and were more often currently working (Table 1). There were significantly more outpatients with a first MDE in the group of responders than in the nonresponders group $(61.0 \%$ versus $50.1 \%, p<0.001)$. The duration of previous MDE was significantly lower in the group of responders (30.43 \pm 22.50 months) compared to the group of non-responders $(34.47 \pm 29.34)$. Finally, the percentage of subjects who attempted suicide was significantly lower in the group of responders (Table 1).

\section{Pharmacogenetics association of CRHR1 with antidepressant response}

A total of 16 SNPs encompassing the four candidate genes, NR3C1, FKPB5, CRHR1, and AVPR1B, were genotyped. The allelic distribution of SNPs was compared between responders and non-responders to identify a pharmacogenetics association to tianeptine antidepressant treatment (Table 2). Two SNPs, rs878886 and rs16940665, located in the same gene CRHR1, showed a statistical significant association with the antidepressant response $(p<0.001)$. Responders were more frequently patients carrying the $\mathrm{C}$ allele of $\mathrm{rs} 878886(\mathrm{OR}=1.26$; $95 \%$ $\mathrm{CI}=[1.11 ; 1.44])$. For the $\mathrm{T}$ allele of rs16940665, the OR associated with antidepressant response was of $1.34(95 \%$ $\mathrm{CI}=[1.13 ; 1.60])$. These associations remained significant after correction for multiple tests ( $r 8878886 p_{\text {corrected }}=$ 0.006 ; rs $16940665 p_{\text {corrected }}=0.013$ ) and a random split of the sample size (Supplementary Table 1).

The pharmacogenetics association confers an attributable risk of response of $16.35 \%$ and $20.32 \%$, respectively, for rs878886*C and rs16940665*T alleles.

Pharmacogenetics analyze were also performed according to the self-report after 2 and 4 weeks of treatment. Only SNP rs878886 of CRHR1 gene showed a significant association with a positive treatment response ( $p=0.019$ at 2 weeks; $p=0.039$ at 4 weeks).

\section{Pharmacogenetic association of CRHR1 with improvement and remission}

We analyzed the pharmacogenetics to remission for tianeptine antidepressant treatment. Again, only the two SNPs located in the CRHR1 gene showed a significant association ( $\mathrm{rs} 878886 \mathrm{Chi}^{2}=8.482, p=0.004$; rs16940665 $\mathrm{Chi}^{2}=6.202, p=0.013$ ) (Supplementary Table 2). The OR with the C allele of rs878886 was of $1.32(95 \% \mathrm{CI}=$ $[1.10 ; 1.60])$ and for the $T$ allele of rs16940665, the OR was of $1.36(95 \% \mathrm{CI}=[1.17 ; 1.73])$. The pharmacogenetics association confers an attributable risk of remission of $19.52 \%$ and $21.29 \%$, respectively, for $\mathrm{rs}^{2} 88886^{*} \mathrm{C}$ and rs $16940665 \% \mathrm{~T}$ alleles. 


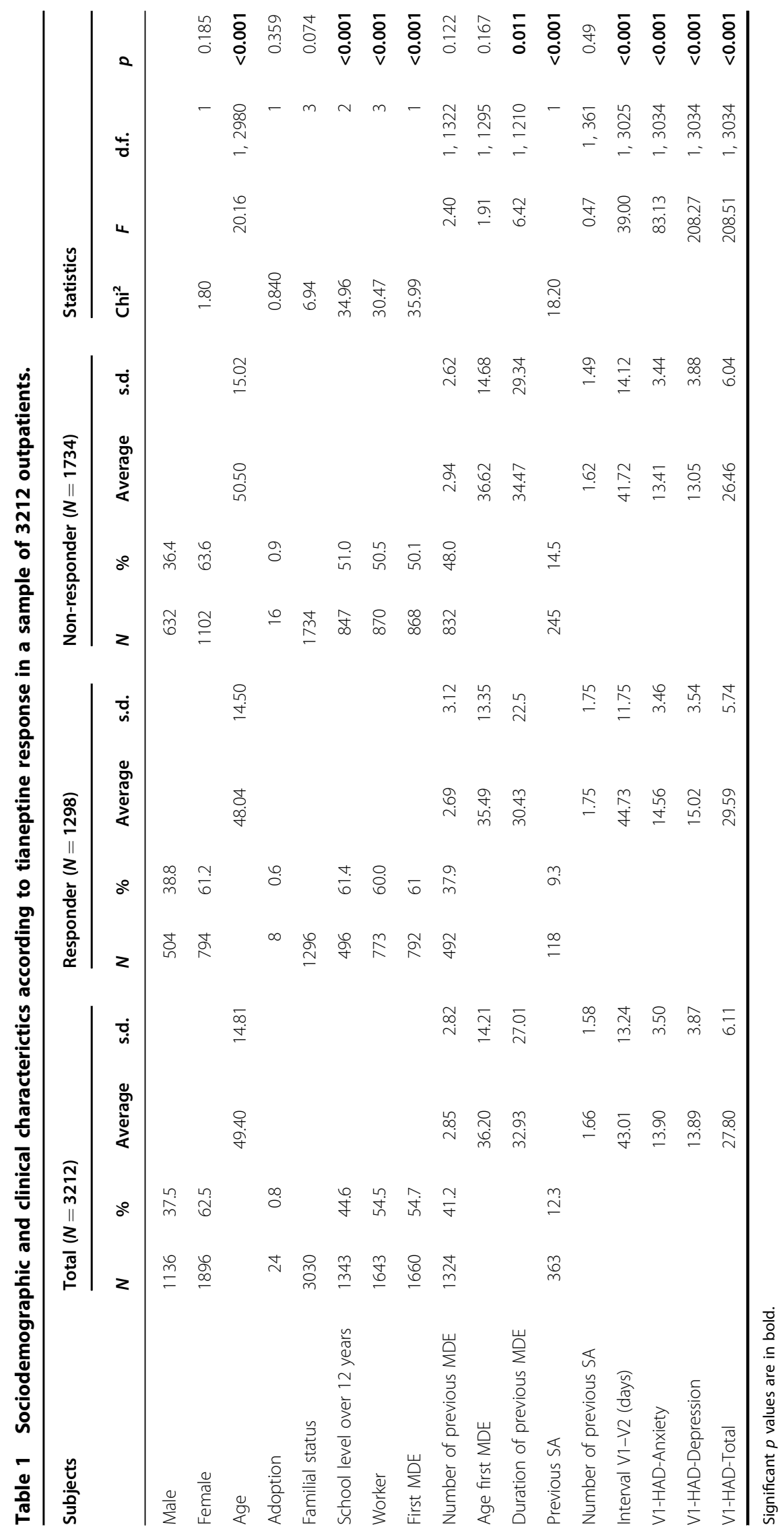


Table 2 Pharmacogenetic association between SNPs of genes regulating the CRF system and response to antidepressant treatment.

\begin{tabular}{|c|c|c|c|c|c|c|c|c|c|}
\hline \multirow[b]{2}{*}{ Gene } & \multirow[b]{2}{*}{ SNPs } & \multirow[b]{2}{*}{ Allele } & \multicolumn{2}{|c|}{ Responders } & \multicolumn{2}{|c|}{ Non-responders } & \multicolumn{3}{|c|}{ Statistics } \\
\hline & & & $N$ & $\%$ & $N$ & $\%$ & $\mathrm{Chi}^{2}$ & d.f. & $p$ \\
\hline \multirow[t]{7}{*}{ NR3C1 } & rs33388 & A & 1123 & 51.2 & 1510 & 52.1 & 0.41 & 1 & 0.520 \\
\hline & rs4912905 & G & 1640 & 75.5 & 2265 & 77.6 & 2.96 & 1 & 0.085 \\
\hline & rs2963155 & A & 1756 & 78.5 & 2266 & 76.6 & 2.71 & 1 & 0.100 \\
\hline & rs41423247 & G & 1433 & 65.7 & 1900 & 65.4 & 0.68 & 1 & 0.794 \\
\hline & rs6189 & G & 2150 & 97.8 & 2907 & 98.0 & 0.24 & 1 & 0.627 \\
\hline & rs4607376 & G & 1170 & 53.4 & 1493 & 50.7 & 3.57 & 1 & 0.059 \\
\hline & rs12656106 & G & 1188 & 54.9 & 1579 & 54.5 & 0.07 & 1 & 0.791 \\
\hline \multirow[t]{6}{*}{ FKBP5 } & rs3800373 & $\mathrm{T}$ & 1488 & 70.1 & 2022 & 71.3 & 0.98 & 1 & 0.322 \\
\hline & rs7757037 & G & 1187 & 54.0 & 1549 & 52.5 & 1.01 & 1 & 0.316 \\
\hline & rs737054 & C & 1610 & 72.3 & 2111 & 71.9 & 0.14 & 1 & 0.706 \\
\hline & rs1360780 & C & 1505 & 68.6 & 2040 & 69.6 & 0.57 & 1 & 0.452 \\
\hline & rs9470080 & C & 1464 & 67.1 & 1970 & 67.8 & 0.28 & 1 & 0.600 \\
\hline & rs6902321 & $\mathrm{T}$ & 1567 & 68.8 & 2093 & 68.4 & 0.12 & 1 & 0.726 \\
\hline \multirow[t]{2}{*}{ CRHR1 } & rs878886 & C & 1710 & 78.2 & 2153 & 73.9 & 12.39 & 1 & $<0.001$ \\
\hline & rs16940665 & $\mathrm{T}$ & 1106 & 79.5 & 1180 & 74.2 & 11.39 & 1 & $<0.001$ \\
\hline AVPR1B & rs28632197 & G & 1885 & 88.7 & 2572 & 89.5 & 0.70 & 1 & 0.402 \\
\hline
\end{tabular}

Significant $p$ values are in bold.

\section{CRHR1 haplotype association with response and remission to antidepressant}

The variants rs878886 and rs16940665 were located in the same haplotype block (linkage disequilibrium of 0.99 and $r^{2}$ of 0.99 ). The frequent haplotype rs878886*C-rs16940665*T was detected in $75.7 \%$ of the subjects. This haplotype was over-represented in $78.1 \%$ of responders compared to $73.9 \%$ of non-responders $\left(\mathrm{Chi}^{2}=12.327, p=4.10-4\right)$. The OR for haplotype rs878886*C-rs16940665*T was of 1.26 (95\% $\mathrm{CI}=[1.11 ; 1.43])$. This haplotype confers an attributable risk of pharmacogenetics response of $16.23 \%$.

The haplotype rs878886*C-rs16940665*T was also significantly over-represented in the remitters (79.8\%) compared to the non-remitters $(75.0 \%)\left(\mathrm{Chi}^{2}=8.559, p=\right.$ $0.003)$, conferring an $\mathrm{OR}=1.32(95 \% \mathrm{CI}=[1.10 ; 1.60])$ and an attributable risk of $19.48 \%$.

Is the involved SNP associated with treatment response through specific clinical features?

To identify an association between SNPs and treatment response through a specific sociodemographic or clinical features we performed logistic regression and ANCOVA. Thus, sociodemographic young age, low school level and worker status, as well as clinical features of a first MDE, young age of the first MDE and high score to the HADS were significantly associated with treatment response (Table 3).

Otherwise, female gender, adoption status, low school level, first MDE, number of previous MDE, lifetime MDE, and previous suicide attempt(s) were associated with CRHR1 SNP rs878886.

Finally, significant interactions in responders and allele $\mathrm{C}$ of $C R H R 1$ rs878886 were observed for young age and first MDE, respectively, $p=0.004$ and $p=0.046$ (Table 3). While we observed a direct and strong effect in the positive treatment response and remission associated with the presence of the allele $C$ of rs878886 or allele $\mathrm{T}$ of rs16940665, we cannot exclude direct and specific effects of sociodemographic status and/or clinical features (Supplementary Fig. 1). Thus, we investigate the simple mediation effect by structural equation models.

Mediation model of joint effect on response to tianeptine of differences in sociodemographic characteristics and depressive disorder features associated with rs8788861

When examining all sociodemographic characteristics and depressive disorder features while mutually adjusting for each other in the same model, individuals with rs8788861 were more likely to be older at the time of the 
Table 3 Logistic regression of socio and clinical traits and their potential role as intermediate variables with CRHR1 SNP rs878886 in predictor for treatment response.

\begin{tabular}{|c|c|c|c|c|c|c|c|c|c|}
\hline \multirow[t]{2}{*}{ Covariable } & \multicolumn{3}{|c|}{$\begin{array}{l}\text { Association with treatment } \\
\text { response }\end{array}$} & \multicolumn{3}{|c|}{$\begin{array}{l}\text { Association with CRHR1 SNP } \\
\text { rs878886 }\end{array}$} & \multicolumn{3}{|c|}{$\begin{array}{l}\text { Interaction with CRHR1 to predict } \\
\text { TTT response }\end{array}$} \\
\hline & Wald & $p$ & OR & $\mathrm{Chi}^{2}$ & $p$ & OR & $\mathrm{Chi}^{2}$ & $p$ & OR \\
\hline Gender: woman & 1.19 & 0.28 & n.a. ${ }^{a}$ & 11.56 & 0.001 & $0.75(0.63-0.88)$ & 1.13 & 0.29 & n.a. \\
\hline Age & 21.73 & $<0.001$ & $1.02(1.01-1.03)$ & 3.33 & 0.068 & $0.65(0.41-1.00)$ & 8.13 & 0.004 & $0.99(0.98-0.99)$ \\
\hline Adoption & 0.97 & 0.33 & n.a. & 12.32 & $<0.001$ & $0.80(0.69-90)$ & 1.15 & 0.28 & n.a. \\
\hline Familial status: not alone & 2.06 & 0.15 & n.a. & 1.06 & 0.30 & n.a. & 0.01 & 0.98 & n.a. \\
\hline School level over 12 years: low & 9.43 & 0.002 & $1.55(1.17-2.05)$ & 7.56 & 0.006 & $0.71(0.56-0.91)$ & 0.03 & 0.87 & n.a. \\
\hline Worker: yes & 9.13 & 0.003 & $0.59(0.42-0.83)$ & 3.55 & 0.06 & $0.72(0.51-1.01)$ & 2.32 & 0.13 & n.a. \\
\hline First MDE & 26.71 & $<0.001$ & $0.54(0.43-0.68)$ & 13.46 & $<0.001$ & $0.69(0.56-0.84)$ & 3.97 & 0.046 & $1.31(1.00-1.71)$ \\
\hline Number of previous MDE & 0.71 & 0.40 & n.a. & 6.30 & 0.012 & $0.69(0.51-0.91)$ & 0.04 & 0.85 & n.a. \\
\hline Age at first MDE & 4.74 & 0.029 & $1.01(1.00-1.03)$ & 0.01 & 0.94 & n.a. & 1.34 & 0.25 & n.a. \\
\hline Lifetime being depressed & 0.36 & 0.55 & n.a. & 6.77 & 0.009 & $0.64(0.46-0.90)$ & 0.60 & 0.44 & n.a. \\
\hline Previous SA & 0.23 & 0.63 & n.a. & 15.95 & $<0.001$ & $0.75(0.65-0.87)$ & 4.56 & 0.03 & $1.60(1.04-2.45)$ \\
\hline Number of previous SA & 1.28 & 0.26 & n.a. & 0.19 & 0.67 & n.a. & 0.02 & 0.88 & n.a. \\
\hline Interval V1-V2 (days) & 6.27 & 0.01 & $0.99(0.98-0.99)$ & 0.16 & 0.69 & n.a. & 2.28 & 0.13 & n.a. \\
\hline V1-HAD-Anxiety & 41.55 & $<0.001$ & $0.89(0.86-0.93)$ & 1.45 & 0.23 & n.a. & 0.20 & 0.65 & n.a. \\
\hline V1-HAD-Depression & 81.68 & $<0.001$ & $0.86(0.83-0.89)$ & 1.20 & 0.27 & n.a. & 0.10 & 0.75 & n.a. \\
\hline V1-HAD-Total & 89.07 & $<0.001$ & $0.90(0.88-0.92)$ & 1.99 & 0.16 & n.a. & 0.68 & 0.41 & n.a. \\
\hline
\end{tabular}

${ }^{a}$ Not applicable.

Significant $p$ values are in bold

study and at the time of the first MDE. The response to antidepressant varied across sociodemographic characteristics and depressive disorder features: low education level, duration of current MDE of more than 6 months, and lifetime suicide attempts having the greater reducing effects on treatment response (Fig. 1).

The total indirect effect (obtained by summing the product of the effects of rs8788861 on each sociodemographic characteristic and depressive disorder feature with their effects on response to antidepressant) was not significant ( $\beta$ $=0.04, \quad p=0.07)$. This non-significant indirect effect implies that differences in prevalence of sociodemographic characteristics and depressive disorder features associated with rs8788861 do not explain the greater response to tianeptine among individuals with rs8788861.

The model also found a significant positive direct effect (i.e., an effect not due to the differences in sociodemographic characteristics and depressive disorder features) of rs8788861 on response to tianeptine (standardized $\beta=0.11, p=0.011$ ), suggesting that the effect of at least certain sociodemographic characteristics and depressive disorder features on response to tianeptine could differ between individuals with and without rs8788861. The sensitivity analysis excluding participants with missing data yielded similar results with a total indirect effect not significant (standardized $\beta=0.01, p=$ 0.675 ) and a significant positive direct effect of rs8788861 (standardized $\beta=0.14, p=0.037$ ) (Supplementary Fig. 2).

The results of this model, particularly the direct effect of rs8788861 on response to tianeptine, suggested that the mediational model did not explain totally the greater response to tianeptine among individuals with rs8788861.

\section{Moderated mediation model of joint effect on response to tianeptine of differences in frequency and effect of sociodemographic characteristics and depressive disorder features associated with rs8788861}

When allowing the effects of sociodemographic characteristics and depressive disorder features to be moderated by rs8788861, there were significant interactions between rs8788861 and age, duration of current MDE higher than 6 months, age at first MDE, and history of suicide attempts. The strongest moderating effect of rs8788861 was on history of suicide attempts and the weakest effects were on sex, working status, and education level (Fig. 2). The total indirect effects in this model was $\beta=0.23$ ( $\mathrm{SE}=0.08, p<0.01)$ and the residual direct effect of rs8788861 was not significant $(\beta=-0.09, \mathrm{SE}=0.09$, $p=0.324$ ), indicating that the total effect of rs8788861 on response to tianeptine (i.e. $\beta=0.15, \mathrm{SE}=0.04$ ) was well explained by this moderated mediation model. The sensitivity analysis excluding participants with missing data yielded similar results, with total indirect effects $(\beta=0.10$, $\mathrm{SE}=0.05, p<0.01)$ and a non-significant residual direct effect of rs8788861 $(\beta=-0.04, \mathrm{SE}=0.18, p=0.835)$ (Supplementary Fig. 3).

Thus, this model indicated that differences in the effects of sociodemographic characteristics and depressive disorder features explained a substantial proportion of differences in response to tianeptine associated with rs8788861. 


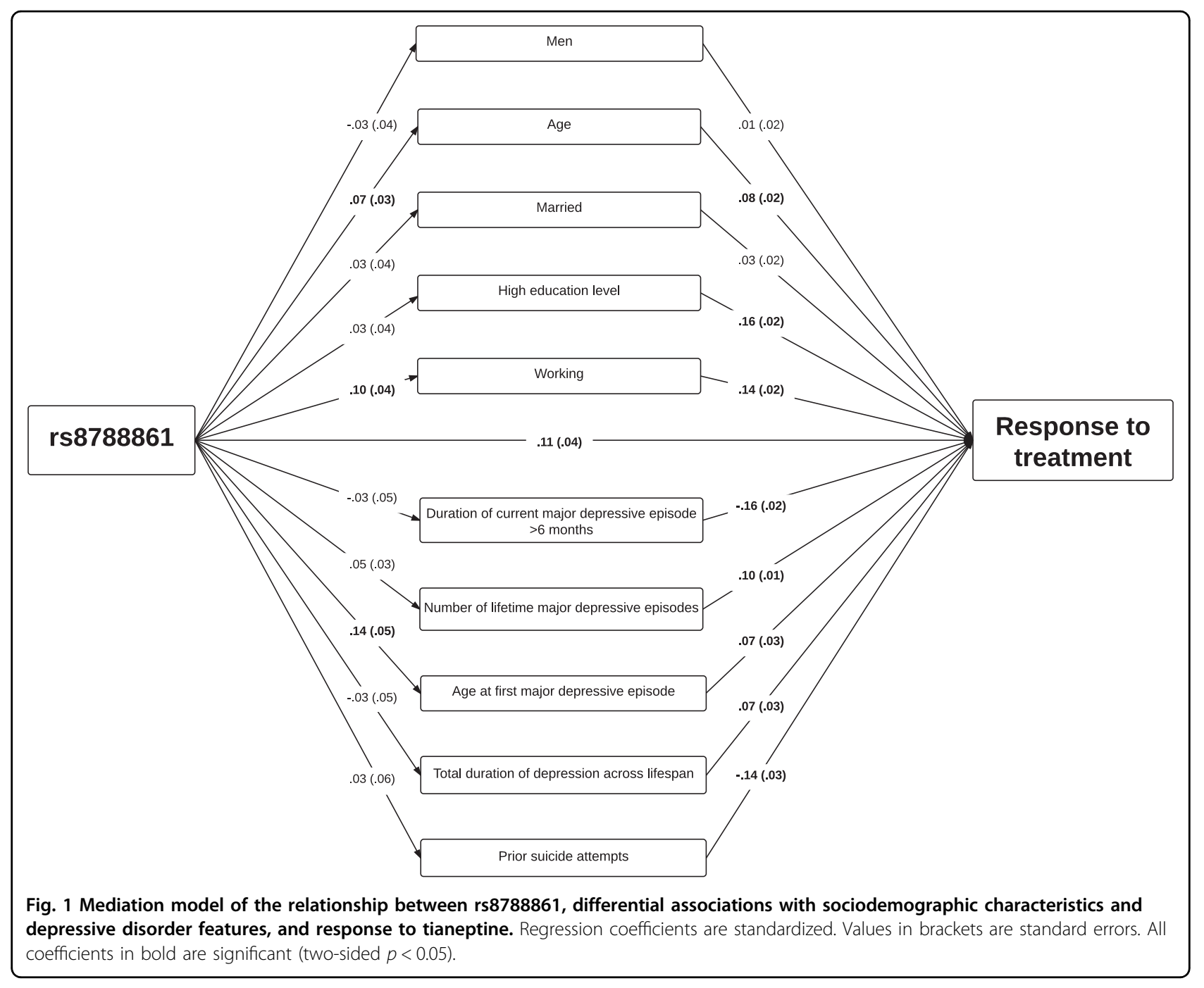

\section{Discussion}

In this study, we have investigated the pharmacogenetics association of multiple variants encompassing four genes involved in the HPA axis, including NR3C1, FKPB5, $C R H R 1$, and $A V P R 1 B$, in a large population of more than 3200 Caucasian outpatients harboring an MDE, recruited and followed up for at least 6 weeks and receiving the atypical antidepressant tianeptine, initiated in $79.5 \%$ of the outpatients and change in $18.8 \%$. Outpatients with additional treatments, including antiparkinsonian drugs, corticosteroids, thyroid hormones, mood stabilizers, antipsychotics, or both, do not have an advantage in treatment response compared to responders without these treatments. Only two polymorphisms within the CRHR1 gene, rs878886 and rs16940665, and haplotype rs $878886 * \mathrm{C}-\mathrm{rs} 16940665 * \mathrm{~T}$ were found significantly associated with a better response to tianeptine, as well as a better remission.
Variants of the CRHR1 gene could be intermediate factors between sociodemographic features or clinical characteristics and treatment response. To avoid this, we recruit outpatients in real life and followed them up for at least 6 weeks, with HADS self-reports at 2- and 4 weeks in addition to HADS reports completed by practitioners at baseline and at the end of follow-up. When sociodemographic features are taken into account, we found common features associated with a better treatment response: a young age, a higher educational level, and a job situation (Table 1). The same observations were done for the clinical characteristics: a first MDE, a lower duration of previous MDE, and less lifetime suicide attempts in the group of responders compared to the nonresponders. When we added the CRHR1 variant rs878886, all these characteristics have less impact in the treatment response and remission than the fact to carry the $\mathrm{C}$ antidepressant responder allele. 


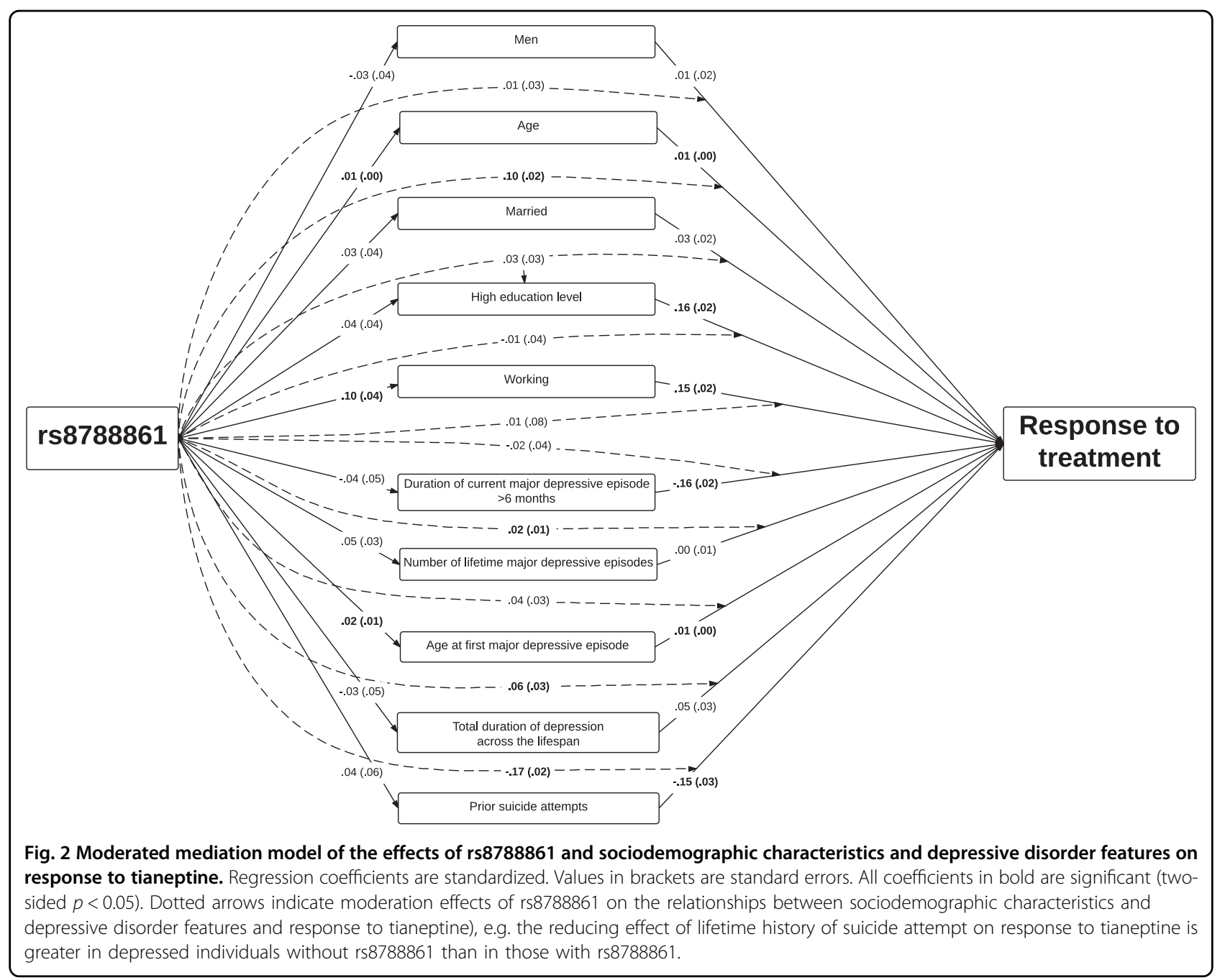

The problem of chance finding is crucial in pharmacogenetic studies according of the numerous discrepancies found in different publications and the possible clinical heterogeneity of the patients, especially these with an MDE. Accordingly, we used three different technics in order to potential reduce this risk. Firstly, we applied the Bonferroni correction although sometimes considered as too conservative, i.e. dividing the $p$ value by the number of SNPs $\left(\right.$ rs $878886 p_{\text {corrected }}=0.006 ;$ rs $16940665 p_{\text {corrected }}=$ 0.013). Secondly, we focused not only on treatment response but also we assessed remission, two concepts that are overlapping but nevertheless not identical (rs878886 $p=0.004 ; \operatorname{rs} 16940665 p=0.013$ ). And lastly, thanks to a large sample size of $3212 \mathrm{MDE}$ outpatients, we randomly split the cohort in two subsamples and confirmed that our results were independently detected (Supplementary Table 1).

Several independent studies have reported pharmacogenetics associations between different polymorphisms of CRHR1 gene with response and remission for several antidepressants, including SSRIs (fluoxetine and citalopram) or a TCA (desipramine) ${ }^{14-16}$. These observations were found in small cohorts of few hundreds to up to two thousands (i.e. STAR*D) depressed patients, from different ethnic origins, and under different antidepressant treatments. In contrast, some other studies did not reported pharmacogenetics association between different CRHR1 polymorphisms and antidepressant treatments $^{29,30}$. Thus, no associations with CRHR1 variants were observed in 159 depressive outpatients treated by citalopram (SSRI) during 4 weeks $^{30}$. Furthermore, the sequencing of CRHR1 in 272 unrelated Mexican Americans with MDE allowed to identify a total of 56 polymorphisms among the gene, including 23 new variants and one that changes the amino acid sequence of the protein. However, none of them were associated with an antidepressant treatment response ${ }^{31}$. Finally, in a 6-week study in duloxetine treated (a serotonin norepinephrine reuptake inhibitor) of $241 \mathrm{MDE}$ patients, only rs4792888 of CRHR1 showed a trend of pharmacogenetics 
association $(p=0.054)^{32}$. These discrepancies could be due to the involvement of different polymorphisms located in other genes in the biological pathway of the HPA axis or they could depend on mechanism of action of antidepressant used.

Thus, polymorphisms in the genes FKBP5 and NR3C1 were found to be associated with antidepressant response in independent studies ${ }^{11-13,17}$. In the present study, we did not observed a pharmacogenetics association for FKBP5 and NR3C1 genes, may be due to the type of antidepressant used or to the outpatients sample. Other variants of genes involved in the HPA axis, including $C R H R 2$ and $C R H B P$, have been reported associated with antidepressant, mostly SSRI or TCA, response or remis$\operatorname{sion}^{15-17}$. In a recent work, the analysis of 636 patients from the International Study to Predict Optimized Treatment in Depression (iSPOT-D), treated 8-week with antidepressants escitalopram (SSRI), sertraline (SSRI), or extended-release venlafaxine (SNRI), have showed a pharmacogenetics association with CRHBP variant rs $28365143^{33}$. Again, discrepancies have been observed between pharmacogenetics association and these genes involved in the biological pathway of the HPA axis.

These divergent observations could be due to different clinical characteristics or different activity of the HPA axis in patients. Thus, it has been reported that fluoxetine (SSRI) responders and remitters exhibited significantly lower circadian cortisol levels than those who did not respond. Furthermore, MDD treated patients who abandoned before the third week presented a low cortisol levels ${ }^{29}$. Several studies have reported that variants in CRHR1 have been associated with specific cortisol responses and stress-system processes ${ }^{32-35}$. While other works have supported that polymorphisms of other genes of CRH axis, like CRHBP gene, affect the cortisol responses $^{36,37}$. Variants in CRHR1 gene have been found associated with several clinical features, like stress, panic, emotion, work memory, and disorders including MDE but also general anxiety disorder ${ }^{37-42}$. Furthermore, these association have been found also in a strong interaction with early life stress and childhood maltreatment ${ }^{41-43}$. In our study, early adverse events have not been collected. Because early adverse events were not collected, we could not assess their differences between responders and nonresponders, which represents a limit of the present work. Interestingly, alleles of rs878886 of CRHR1 gene have been associated with fear acquisition in humans ${ }^{44,45}$. These studies showed that G-allele carriers have a reduced acquisition of cue-specific fear-conditioned responses compared to $\mathrm{C} / \mathrm{C}$ homozygotes. The cuedfear acquisition deficits is also under the genetic interaction of variations of the SL6A4/5HTT gene, which codes for the serotonin transporter ${ }^{44,45}$. This fear acquisition under the control of CRHR1 and SLC6A4 variants may be also a limitation of this study due to the impact of fear control between the responders and nonresponders. These implications, direct or indirect, of CRHR1 variants in the clinical features and disorders, in interaction with life events, can modulate the clinical phenotypes of the MDE subjects. And these CRHR1 variants can also impact, directly or indirectly, antidepressant treatment response.

Here, we investigate the pharmacogenetics treatment response of the atypical antidepressant tianeptine. Tianeptine enhances a cascade of cellular adaptations that prevents and reverses the dendritic remodeling induced by the stress or glucocorticoids and increases dendritic length and branching of neurons in the amygdala ${ }^{19,20}$. Interestingly, it has been showed that serotonin from the dorsal raphe nucleus enhances fear and anxiety and activates a subpopulation of corticotropin-releasing factor neurons in the bed nucleus of the stria terminalis ${ }^{46}$. This circuit could be modulated by tianeptine to improve a better adaptation, in contrast to the clinical observations of early adverse events to SSRI treatment in some patients. Another propriety of tianeptine is its MOR agonist that acts specifically on the opioidergic system ${ }^{22}$. Indeed, a recent study has shown, in mice model, that the antidepressant- and opioidlike behavioral effects of tianeptine were mediated by its MOR agonist ${ }^{22}$. Interestingly, cortisol response is modulated by the endogenous opioid, beta-endorphin, via the MOR and variant in the MOR gene OPRM1 affects the cortisol stress response ${ }^{23}$ and, as mentioned previously, opioid system partly regulates HPA axis activation.

To conclude, our study was able to strongly replicate a pharmacogenetics association of an atypical antidepressant tianeptine treatment for MDE, with one of the gene involved in the HPA axis, CRHR1 gene. This pharmacogenetics work shows CRHR1 association with (1) more specifically attributed to treatment response, (2) with a lower risk of chance finding, and (3) in a more ecological situation. Our results should encourage studies on the link between opioid system and HPA axis. Furthermore, recent findings on ketamine support an interaction between these systems. Indeed, it has been shown that ketamine increases the effectiveness of the signaling induced by opiates ${ }^{47}$ and in a very recent randomized controlled trial ${ }^{48}$, they found that reduction of depression was lower when naltrexone (an MOR antagonist), versus placebo, was administered before ketamine administration. Regarding those data and results of our study, it seems that there is a real interaction between HPA and opioid systems and this interaction may modulate response to antidepressant treatment and evolution of depression.

\section{Acknowledgements}

We thank all the outpatients and clinicians who participated in this national study. 


\section{Author details}

'Université de Paris, Institute of Psychiatry and Neuroscience of Paris (IPNP), INSERM U1266, Team Vulnerability of Psychiatric and Addictive Disorders, 75014 Paris, France. ${ }^{2}$ Assistance Publique-Hôpitaux de Paris (APHP), Corentin Celton Hospital, Department of Psychiatry, 92130 Issy-les-Moulineaux, France. ${ }^{3}$ University of Paris, Paris, France. ${ }^{4}$ Department of Emergency Psychiatry and Acute Care, CHU Montpellier, INSERM U1061, Montpellier University, Montpellier, France. ${ }^{5} \mathrm{GHU}$ Paris Psychiatrie et Neurosciences, Clinique des Maladies Mentales et de l'Encéphale (CMME), Centre Hospitalier Sainte-Anne, Paris, France. ${ }^{6}$ Service de Psychiatrie, Hôpital Louis Mourier, Assistance Publique-Hôpitaux de Paris, Colombes, France

\section{Author contributions}

P.G. and P.C. designed the study. N.R., P.G., Y.L.S. and P.C. followed up the acquisition of the data. N.R., N.H., B.N., G.V. and A.N. analyzed and interpreted the data. N.R., P.G., N.H., B.N., G.V. and P.C. wrote and revised the manuscript. All approved the final version of the paper.

\section{Conflict of interest}

The present work was supported by a research grant from Servier, who had no involvement in the design, organization, analysis, or preparation for publication of the study. N.R., N.H., B.N., G.V., Y.L.S., and A.N. have no conflicts of interest to declare. P.C. has received fees for his participation on the advisory board from Servier during the last 5 years. P.G. received during the last 5 years fees for presentations at congresses or participation in scientific boards from Alcediag-Alcen, Angelini, GSK, Janssen, Lundbeck, Otsuka, SAGE, and Servier.

\section{Publisher's note}

Springer Nature remains neutral with regard to jurisdictional claims in published maps and institutional affiliations.

Supplementary Information accompanies this paper at (https://doi.org/ 10.1038/s41398-020-01067-y).

Received: 11 March 2020 Revised: 29 September 2020 Accepted: 19 October 2020

Published online: 05 November 2020

\section{References}

1. Kohler, O., Krogh, J., Mors, O. \& Benros, M. E. Inflammation in depression and the potential for anti-inflammatory treatment. Curr. Neuropharmacol. 14, 32-42 (2016).

2. Bissette, G., Klimek, V., Pan, J., Stockmeier, C. \& Ordway, G. Elevated concentrations of CRF in the locus coeruleus of depressed subjects. Neuropsychopharmacology 28, 1328-1335 (2003).

3. Nemeroff, C. B., Owens, M. J., Bissette, G., Andorn, A. C. \& Stanley, M. Reduced corticotropin releasing factor binding sites in the frontal cortex of suicide victims. Arch. Gen. Psychiatry 45, 577-579 (1988).

4. Müller, M. B. et al. Limbic corticotropin-releasing hormone receptor 1 mediates anxiety-related behavior and hormonal adaptation to stress. Nat. Neurosci. 6, 1100-1107 (2003).

5. Fries, G. R., Gassen, N. C. \& Rein, T. The FKBP51 glucocorticoid receptor cochaperone: regulation, function, and implications in health and disease. Int. J. Mol. Sci. 18, E2614 (2017).

6. De Maricourt, P., Jay, T., Goncalvès, P., Lôo, H. \& Gaillard, R. Ketamine's antidepressant effect: literature review on clinical use. Encephale 40, 15-23 (2014).

7. Chen, M. H. et al. Rapid inflammation modulation and antidepressant efficacy of a low-dose ketamine infusion in treatment-resistant depression: a randomized, double-blind control study. Psychiatry Res. 269, 207-211 (2018).

8. De Bellis, M. D., Gold, P. W., Geracioti, T. D. Jr, Listwak, S. J. \& Kling, M. A. Association of fluoxetine treatment with reductions in CSF concentrations of corticotropinreleasing hormone and arginine vasopressin in patients with major depression. Am. J. Psychiatry 150, 656-657 (1993).

9. Kling, M. A. et al. Effects of electroconvulsive therapy on the CRH-ACTHcortisol system in melancholic depression: preliminary findings. Psychopharmacol. Bull. 30, 489-494 (1994).
10. Heuser, I. J. et al. Pituitary-adrenal-system regulation and psychopathology during amitriptyline treatment in elderly depressed patients and normal comparison subjects. Am. J. Psychiatry 153, $93-99$ (1996).

11. Pariante, C. M. \& Miller, A. H. Glucocorticoid receptors in major depression: relevance to pathophysiology and treatment. Biol. Psychiatry 49, 391-404 (2001).

12. Binder, E. B. et al. Polymorphisms in FKBP5 are associated with increased recurrence of depressive episodes and rapid response to antidepressant treatment. Nat. Genet. 36, 1319-1325 (2004)

13. van Rossum, E. F. et al. Polymorphisms of the glucocorticoid receptor gene and major depression. Biol. Psychiatry 59, 681-688 (2006).

14. Uher, R. et al. Genetic predictors of response to antidepressants in the GENDEP project. Pharmacogenomics. J. 9, 225-233 (2009).

15. Licinio, J. et al. Association of a corticotropin-releasing hormone receptor 1 haplotype and antidepressant treatment response in Mexican-Americans. Mol. Psychiatry 9, 1075-1082 (2004).

16. Binder, E. B. et al. Association of polymorphisms in genes regulating the corticotropin-releasing factor system with antidepressant treatment response. Arch. Gen. Psychiatry 67, 369-379 (2010).

17. Voegeli, G., Cléry-Melin, M. L., Ramoz, N. \& Gorwood, P. Progress in elucidating biomarkers of antidepressant pharmacological treatment response: a systematic review and meta-analysis of the last 15 years. Drugs 77, 1967-1986 (2017).

18. Gorwood, P. \& Hamon, M. (eds.). Psychopharmacogenetics (Springer Science, New York, 2006).

19. Magarinos, A. M. \& McEwen, B. S. Stress-induced atrophy of apical dendrites of hippocampal CA3C neurons: involvement of glucocorticoid secretion and excitatory amino acid receptors. Neuroscience 69, 89-98 (1995).

20. Watanabe, Y., Gould, E., Daniels, D. C., Cameron, H. \& McEwen, B. S. Tianeptine attenuates stress-induced morphological changes in the hippocampus. Eur. J. Pharmacol. 222, 157-162 (1992).

21. Gassaway, M. M., Rives, M. L., Kruegel, A. C., Javitch, J. A. \& Sames, D. The atypical antidepressant and neurorestorative agent tianeptine is a $\mu$-opioid receptor agonist. Transl. Psychiatry 4, e411 (2014).

22. Samuels, B. A. et al. The behavioral effects of the antidepressant tianeptine require the mu-opioid receptor. Neuropsychopharmacology 42, 2052-2063 (2017).

23. Drolet, G. et al. Role of endogenous opioidsystem in the regulation of the stress response. Prog. Neuropsychopharmacol. Biol. Psychiatry 25, 729-741 (2001).

24. Lovallo, W. R. et al. Cortisol stress response in men and women modulated differentially by the mu-opioid receptor gene polymorphism OPRM1 A118G. Neuropsychopharmacology 40, 2546-2554 (2015).

25. McEwen, B. S. et al. The neurobiological properties of tianeptine (Stablon): from monoamine hypothesis to glutamatergic modulation. Mol. Psychiatry 15, 237-249 (2010).

26. Gorwood, P., Corruble, E., Falissard, B. \& Goodwin, G. M. Toxic effects of depression on brain function: impairment of delayed recall and the cumulative length of depressive disorder in a large sample of depressed outpatients. Am. J. Psychiatry 165, 731-739 (2008).

27. Gorwood, P. et al. Is it worth assessing progress as early as week 2 to adapt antidepressive treatment strategy? Results from a study on agomelatine and a global meta-analysis. Eur. Psychiatry 28, 362-371 (2013).

28. Friedman, S., Samuelian, J. C., Lancrenon, S., Even, C. \& Chiarelli, P. Threedimensional structure of the Hospital Anxiety and Depression Scale in a large French primary care population suffering from major depression. Psychiatry Res. 104, 247-257 (2001).

29. Ventura-Juncá, R. et al. Relationship of cortisol levels and genetic polymorphisms to antidepressant response to placebo and fluoxetine in patients with major depressive disorder: a prospective study. Bmc. Psychiatry 14, 220 (2014).

30. Papiol, S. et al. Genetic variability at HPA axis in major depression and clinical response to antidepressant treatment. J. Affect. Disord. 104, 83-90 (2007).

31. Dong, C., Wong, M. L. \& Licinio, J. Sequence variations of ABCB1, SLC6A2, SLC6A3, SLC6A4, CREB1, CRHR1 and NTRK2: association with major depression and antidepressant response in Mexican-Americans. Mol. Psychiatry 14, 1105-1118 (2009).

32. Perlis, R. H., Fijal, B., Dharia, S. \& Houston, J. P. Pharmacogenetic investigation of response to duloxetine treatment in generalized anxiety disorder. Pharmacogenomics. J. 13, 280-285 (2013). 
33. O'Connell, C. P. et al. Antidepressant outcomes predicted by genetic variation in corticotropin-releasing hormone binding protein. Am. J. Psychiatry 175 251-261 (2018).

34. Sumner, J. A., McLaughlin, K. A., Walsh, K., Sheridan, M. A. \& Koenen, K. C. CRHR1 genotype and history of maltreatment predict cortisol reactivity to stress in adolescents. Psychoneuroendocrinology 43, 71-80 (2014).

35. Pagliaccio, D. et al. Stress-system genes and life stress predict cortisol levels and amygdala and hippocampal volumes in children. Neuropsychopharmacology 39, 1245-1253 (2014).

36. Davis, E. G. et al. Corticotropin-releasing factor 1 receptor haplotype and cognitive features of major depression. Transl. Psychiatry 8, 5 (2018).

37. Mahon, P. B., Zandi, P. P., Potash, J. B., Nestadt, G. \& Wand, G. S. Genetic association of FKBP5 and CRHR1 with cortisol response to acute psychosocial stress in healthy adults. Psychopharmacology 227, 231-241 (2013).

38. Schatzberg, A. F. et al. HPA axis genetic variation, cortisol and psychosis in major depression. Mol. Psychiatry 19, 220-227 (2014).

39. Fuge, P. et al. Interaction of early life stress and corticotropin-releasing hormone receptor gene: effects on working memory. Biol. Psychiatry 76, 888-894 (2014).

40. Weber, $\mathrm{H}$. et al. Allelic variation in CRHR1 predisposes to panic disorder: evidence for biased fear processing. Mol. Psychiatry 21, 813-822 (2016).
41. Bradley, R. G. et al. Influence of child abuse on adult depression: moderation by the corticotropin-releasing hormone receptor gene. Arch. Gen. Psychiatry 65, 190-200 (2008).

42. Polanczyk, G. et al. Protective effect of CRHR1 gene variants on the development of adult depression following childhood maltreatment: replication and extension. Arch. Gen. Psychiatry 66, 978-985 (2009).

43. Tyrka, A. R. et al. Interaction of childhood maltreatment with the corticotropinreleasing hormone receptor gene: effects on hypothalamic-pituitary-adrenal axis reactivity. Biol. Psychiatry 66, 681-685 (2009).

44. Heitland, I., Goenink, L., Bijlsma, E. Y., Oosting, R. S. \& Baas, J. M. P. Human fear acquisition deficits in relation to genetic variants of the corticotropin releasing hormone receptor 1 and the serotonin transporter. PLOS ONE 8, e63772 (2013).

45. Heitland, I. et al. Human fear acquisition deficits in relation to genetic variants of the corticotropin-releasing hormone receptor 1 and the serotonin transporter-revisited. Genes Brain Behav. 15, 209-220 (2016).

46. Marcinkiewcz, C. A. et al. Serotonin engages an anxiety and fear-promoting circuit in the extended amygdala. Nature 537, 97-101 (2016).

47. Gupta, A., Devi, L. A. \& Gomes, I. Potentiation of $\mu$-opioid receptor-mediated signaling by ketamine. J. Neurochem. 119, 294-302 (2011).

48. Williams, N. R. et al. Attenuation of antidepressant effects of ketamine by opioid receptor antagonism. Am. J. Psychiatry 175, 1205-1215 (2018). 